\title{
Discrete-State Photomechanical Actuators
}

Amir Alipour Skandani ${ }^{1}$, Sourav Chatterjee ${ }^{1}$, Matthew L. Smith ${ }^{2}$, John Baranski ${ }^{2}$, David H. Wang $^{3}$, Loon-Seng Tan ${ }^{4}$, Timothy J. White ${ }^{4}$ and M. Ravi Shankar ${ }^{1 *}$

${ }^{1}$ Department of Industrial Engineering

3700 O'Hara Street

University of Pittsburgh

Pittsburgh, PA 15261

${ }^{2}$ Department of Engineering

Hope College

Holland, MI 49423, USA

${ }^{3}$ Biological and Nanoscale Technologies Division

UES Inc.

Dayton, OH 45432, USA

${ }^{4}$ Air Force Research Laboratory

Materials and Manufacturing Directorate

Wright Patterson Air Force Base, OH 45433, USA

E-mail: ravishm@pitt.edu, timothy.white.24@us.af.mil 


\begin{abstract}
Directly transducing light into work is attractive for remotely powering soft mechanisms fabricated from photoresponsive polymers, but presents challenges for achieving reliable actuation within a control framework. Here, we utilize azobenzene-functionalized polyimides to fabricate actuators characterized by mechanically discrete states. Irradiation initiates the photochemically induced, quasistatic deformation to advance the actuator to the edge of instability. Following this latency, ultrafast snap through actuation ( 10ms-scale) ensues. Restricting the role of control to the attainment of the edge of instability, strategies for achieving repetitive actuation via multiplexed irradiation are demonstrated. Approaches are examined for modulating the latency of the actuator using an all-optical strategy as well as mechanical design of the actuator. Prototypical assemblies of these actuators in arrays are used to fabricate morphable surfaces and structures, which is aided by the realization that the ultrafast actuation is characterized by a high power-density on the order of $\sim \mathrm{kW} / \mathrm{m}^{3}$.
\end{abstract}




\section{Introduction}

Polymers that are responsive to stimuli (e.g. electrical, thermal, etc.) have been explored as artificial muscles for new classes of soft actuators and robotic systems ${ }^{1-3}$. The design space to use active materials is expanded by the ability to transduce radiant energy into mechanical actuation, especially for miniaturized soft machines, by eliminating the need for onboard power sources. Photoinduced, isothermal mechanical deformation presents opportunities for remote, wireless triggering of responsive structures and reconfigurable surfaces. Polymers that respond via photochemical transformations (e.g. photoisomerization of azobenzene ${ }^{4,5}$ ) have attracted significant interest for their light controlled mechanical adaptivity. Responses in azobenzene-functionalized liquid crystal polymers can be hard-coded by manipulating local director orientations ${ }^{6,7}$ and the crosslinker concentration ${ }^{8}$. In azobenzene-functionalized polyimides, tailoring the backbone rigidity influences the actuation rate and the persistence of the photo-strains ${ }^{9}$. Across the photoresponsive systems, controlling the intensity and polarization of the actinic light is a tool for modulating the actuation ${ }^{4}$. While, high irradiation intensities $\gg>100 \mathrm{~mW} / \mathrm{cm}^{2}$ can induce ultrafast actuation via the superposition of photochemical and photothermal effects, the coupled temperature rise and the concomitant material degradation limits its utility ${ }^{10}$. Photo actuation in azobenzenefunctionalized polymers results from the superposition of non-linear absorption, statistical accumulation of the trans/cis-forms of azobenzene and the gradient of these effects through the thickness of the sample ${ }^{11}$. A range of system designs have been realized using photoresponsive polymers as soft actuators. These include, microvalves ${ }^{12}$ and pumps ${ }^{13}$ fabricated from azobenzene-functionalized liquid crystalline polymers that can be actuated with light, as well as prototypical light-operated robotic systems ${ }^{14,15}$. The latter implementation used multimaterial constructs to create light-powered manipulators. Drawing from explorations of the photomechanical adaptivity as a function of polarization, intensity 
and wavelength, most device implementations have exploited the continuous range (magnitude) of light induced deformations in a range of "analog" mode devices. While this mode of actuation has intrinsic advantages in the spatial granularity of actuation that can be achieved, the utilization of light as the trigger presents intrinsic challenges in the feedback control of the photomechanical actuators. Furthermore, the gradual and monotonic nature of the deformation following irradiation limits the achievable power and impulse that can be generated for driving mechanical systems.

The authors showed the applicability of the exploitation of mechanical instabilities to toggle between binary mechanical states via isothermal, photoisomerization in azobenzenefunctionalized polymers in the earlier work ${ }^{16}$. However, depletion of isomerizable moieties from multiple actuation cycles resulted in the sample becoming unresponsive after 4-5 cycles. The premise of this work is that utilizing a multiplexed irradiation of mechanical designs fabricated from azobenzene-functionalized polyimides can modulate the approach to the photomechanical instability, control the latency of switching and achieve toggling between the discrete states over multiple cycles to enable their deployment in prototypical light controlled mechanical devices. Note that utilizing mechanical bistability simplifies the control loop to one of calibrating the irradiation required for inducing the ultrafast transition between the states. The digitization of the mechanical states is advantageous because the actuator can persist in a given state without requiring any additional power. Indeed, such limits the degrees of freedom of the actuator to discrete states. We demonstrate that this limitation can be ameliorated by utilizing superlattices of the actuators to multiply the number of achievable states. 


\section{Materials and methods}

\subsection{Azopolyimide synthesis and film preparation}

The cross-linked azobenzene-functionalized polyimide (designated as X-ppp-6FDI) is synthesized from a reaction mixture ( 20 wt/v \%, concentration) of $100 \mathrm{~mol} \%$ of 1,1,1,3,3,3hexafluoro-2,2-bis(4-phthalic anhydride)propane (6FDA, MW $=444.24 \mathrm{~g} / \mathrm{mol}), 70 \mathrm{~mol} \%$ of 1,4-bis(4-aminophenoxy)benzene $(\mathrm{MW}=292.33 \mathrm{~g} / \mathrm{mol})$, and $20 \mathrm{~mol} \%$ of azobenzene triamine crosslinker $\quad(4,4$ ', 4 "-[ethylidynetris(4,1-phenyleneoxy-4,1-phenylene-2,1diazenediyl)] tris-benzenamine, MW=892.02 g/mol), following our previously reported twostep procedure ${ }^{17}$. The experimental details for the synthesis of the tris(azobenzene-amine) crosslinker have been previously described in our report ${ }^{9}$.

The condensed chemical structure for X-ppp-6FDI is shown below:

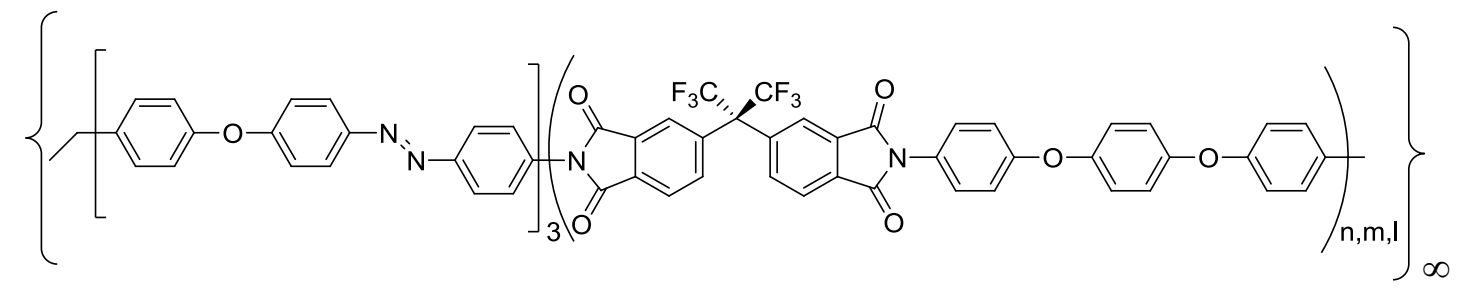

where the azobenzene-containing tri-linked netpoint is represented in square brackets, the linear segments with varied degree of polymerization (DP $=n, m, 1)$ is represented in the parentheses, and the overall network architecture is indicated by the infinity symbol.

In a typical preparation, $100 \mathrm{~mol} \%$ of $6 \mathrm{FDA}$ and $70 \mathrm{~mol} \%$ of 1,4-bis(4aminophenoxy)benzene was in N,N-dimethylacetamide (DMAc) in a nitrogen atmosphere at ambient temperature for $24 \mathrm{~h}$. This generated poly(amic acid) oligomers (PAA) terminated by anhydride groups. Then, $20 \mathrm{~mol} \%$ of azobenzene triamine crosslinker was added. The resulting PAA sol-gel precursor was poured onto $2 " \mathrm{x} 2$ " and $4.5 " \times 4.5 "$ glass slides and cured in an oven, with stepwise heating according to the following schedule: $100{ }^{\circ} \mathrm{C} / 2 \mathrm{~h}, 150$ ${ }^{\circ} \mathrm{C} / 2 \mathrm{~h}, 175^{\circ} \mathrm{C} / 1 \mathrm{~h}, 200{ }^{\circ} \mathrm{C} / 2 \mathrm{~h}, 250{ }^{\circ} \mathrm{C} / 1 \mathrm{~h}$ and $300{ }^{\circ} \mathrm{C} / 1 \mathrm{~h}$, which allowed the polymer films to be 
imidized. Subsequently, the thermally cured films were cooled under identical conditions under the flow of nitrogen to limit the effect of thermal history.

\subsection{Imaging setup and image processing}

The optical setup used was similar to our previous study on snap-through instability, where arch-shaped samples fabricated from photoresponsive materials can be irradiated using lasers of various wavelengths, intensities and polarizations ${ }^{16}$. Samples were held rigidly at their ends between aluminum plates mounted on positioning stages. Translating the ends creates a buckled arch, which is bistable. A range of geometries were created and illuminated with polarized light using a 445nm laser (WickedLasers) and a $375 \mathrm{~nm}$ UV laser (Thorlabs). Samples with both uniform and varying width were used to create these arches. In addition, longer strips were prepared and utilized in a new bifurcated rod mechanism with a constraining rod positioned in the middle. This mechanism was examined for achieving bidirectional photo-induced switching using irradiation from the same direction. The laser power incident on the sample was measured using a digital power and energy meter (Thorlabs). Commercially available optical components (Thorlabs and Newport) were used for polarizing, and illuminating the sample from the top and bottom. Samples were illuminated using a fiber-optic illuminator (Cole Parmer) and imaged using an Aven Mighty Scope camera at 7 frames/sec. When needed, high-speed imaging using a charge-coupled device camera system was used. For analysis, cropped image of the cantilever/arch was preprocessed, using Gaussian smoothing, to remove image noise. A vertical scan for local maximum intensity was used to determine the cantilever shape, which was validated with visual inspection in each case. A polynomial curve was fitted to the cantilever (or arch) profile, and the curvature was calculated from local derivatives of the polynomial fit. 


\section{Results and discussion}

A simple binary actuator is the buckled arch, which is illustrated in Figure 1a. This arch can be in one of the two stable states - arched upward or downward and remains in that state without requiring any external power or impulse. Irradiating such an arch fabricated from a photoresponsive material can deform it quasistatically to reach an instability, after which snap-through instability ensues to achieve the other stable position. While, the snapthrough is ultrafast $(\sim 10 \mathrm{~ms})$, the achievement of the configuration at the edge of instability involves a finite latency and is dependent on the irradiation intensity and the photosensitivity of the material. The time to trigger the snap-through via the quasistatic photomechanical deformation is typically several second. In such a mechanism, the role of the control framework is limited up to the point of the inducement of the snap-through at the edge of instability. After this, the switching of the states is autonomous and occurs in an ultrafast manner with a large mechanical power output.

Currently, the utility of this approach for amplifying photomechanical effects is limited by the build-up of a critical concentration of isomerized chromophores that can render the sample inert and prevent repetitive switching between the states. That is, when the actuator is irradiated, it deforms to reach the edge of instability and then undergoes snapthrough. However, this reduces the subsequent photoresponsiveness with the loss of isomerizable chromophores, which limits the number of actuation cycles. This progressive decrease of sensitivity limits the actuation to four to five discrete snap-through cycles in azobenzene-functionalized polyimides (azo-polyimide). Figure 1b illustrates the strategy for achieving repetitive switching between the bistable states of the simple arch geometry. Rectangular samples of azo-polyimide $\left(L_{0}=14 \mathrm{~mm}, W=1 \mathrm{~mm}, T=15 \mu \mathrm{m}\right)$ were clamped at two ends. The ends are compressed slightly $\left(L_{o}-L=\Delta L=45 \mu \mathrm{m}\right)$ and the strips buckle and form an arch with length $L$ (Figure 1a). Irradiation from a 375nm UV laser with polarization 
set parallel to the long axis of the arch induces actuation. The diameter of the laser beam is $5 \mathrm{~mm}$ and it is focused at the center of the arch. The initial deformation occurs quasistatically for the first $t_{c r}$ seconds (Figure 1b). The accumulation of the contractile strains at the irradiated surface occurs via the trans-cis isomerization of the azobenzene chromophores leading to a distortion of the geometry of the arch. Following latent time of $t_{c r}$, the sample reaches the edge of instability and snap-through ensues to invert the arch. This transition is ultrafast and occurs over $\sim 10 \mathrm{~ms}$ (See supplementary movie 1 ). For an irradiation intensity of $0.2 \mathrm{~mW} / \mathrm{cm}^{2}$ $t_{c r}$ is $\sim 94 \mathrm{~s} . t_{c r}$ is found to monotonically decrease with increasing intensity of UV (Figure 1c). The energy that is accumulated quasi-statically during the approach to instability is released during snap-through. By characterizing the geometry just before and after snap-through we estimate the energy released during the ultrafast actuation. For this estimate, the curvatures were measured $\left(\kappa_{i}\right)$ at $i$ discrete points along the sample. The energy before and after snapthrough is calculated as: $U=\sum_{i} \frac{E I}{2}{ }_{i}^{2}$, where the modulus is $\mathrm{E}=2.77 \mathrm{GPa}^{17}, I$ is the moment of area of the rectangular cross section, and the interval $\Delta$ is the length of discrete segments over which the summation is performed. The difference between the two states for geometry in Figure 1b was found to be $7.2 \mathrm{~nJ}$, which corresponds to a specific power of 3.5 $\mathrm{kW} / \mathrm{m}^{3}$ for the ultrafast actuation that occurs in $10 \mathrm{~ms}$.

After inducing snap-through, the irradiated surface is exposed to $>500 \mathrm{~nm}$ broadband irradiation from a filtered halogen lamp for 300 seconds. This irradiation reverts the conformational form of azobenzene to the trans state via the cis-trans back-reaction ${ }^{4}$ and relaxes the photostrains (Figure 1b). Subsequently, the actuator is irradiated from the bottom in Figure $1 \mathrm{~b}$ to restore it back to the prior state and the photo-responsiveness can again be restored using $>500 \mathrm{~nm}$ irradiation. Such multiplexed irradiation allows for the sample to be repetitively actuated. The potential for repeated toggling of the states of an actuator using 
light is crucial for the deployment of this actuator in an array of applications. Figure 1d illustrates the variation of the normalized latency $\left(t_{c r}\right)$ for multiple actuation cycles. Each actuation cycle is characterized by irradiation with the $375 \mathrm{~nm}$ UV laser with $25 \mathrm{~mW} / \mathrm{cm}^{2}$. After inducing snap through, the irradiation with $>500 \mathrm{~nm}$ light was performed. We find that the restoration of photoresponsive allowed for multiple actuation cycles. Also we find that the very first actuation involved the largest latency. All subsequent cycles involved a substantially reduced latency. The underlying mechanism is unclear and ongoing work is to resolve that.

\subsection{Photoactuated mechanisms}

Figure 2 illustrates prototypes that exploit the ability to achieve multiple actuation cycles in photomechanically controlled bistable actuators. Figure 2 a illustrates a 3 x 3 array of photo-responsive bistable actuators. Using indexed irradiation on the actuators, we demonstrate a customizable topography that can be tailored without contact. For illustrative purposes, we spell-out the letters $\mathrm{P}$, I and T by toggling the actuators appropriately. The actuators that are arched upward are marked green and those arched downward are marked red. It is possible to scale-up this design to significantly larger areas and create surfaces with tailorable topographies that are useful as light-driven micro-mirror arrays, variable reflectivity/topographical morphing, or for contactlessly manipulating drag on flight-control surfaces. Figure $2 \mathrm{~b}$ illustrates a tripod-like structure that utilizes bistable actuators as its three legs. Toggling these actuators individually or in combination allows for reorienting the normal vector of the "table" that rests on them. This system can be considered as a unit-cell of a "binary robotic system" 18 that is composed of arrays of these cells that are stacked on each other. In such a configuration, the number of geometric configurations scales as a power of the number of unit cells and allows for the creation of photo-morphable 3D structures ${ }^{19}$. In these applications, it is possible to circumvent challenges that can arise from occlusion of the optical path by activating the bistable devices using fiber optic elements. Figure S1 and 
Supplementary Movie 2 illustrate the actuation of a bistable actuator using UV irradiation from an optical fiber. This can allow for the creation of 3-dimensional arrays of these actuators to engineer photomechanical devices with multiple degrees-of-freedom, even though individually they are only characterized by two discrete states.

\subsection{Bidirectional photoactuation using unidirectional irradiation}

The utilization of the bistable arch for to-and-fro actuation requires irradiation from the top and the bottom, which can complicate its utilization in systems where the geometry is occluded from either side. Achieving bidirectional actuation using irradiation from the same side is enabled using the design illustrated in Figure 3. Here, a polyimide arch is rigidly held at the ends and is deformed by a line contact with a cylindrical support placed midway between the ends. The idea is to first create a bistable arch, which is then bifurcated at the middle (dashed line in Figure 3a). The resulting asymmetric geometry can be either skewed to the left or the right. Thus, the region between the rigid support and the central support is either arched up or arched down. In Figure 3b, a rectangular strip of the azobenzene-functionalized polyimide $\left(L_{0}=55.5 \mathrm{~mm}, W=1 \mathrm{~mm}, T=15 \mu \mathrm{m}\right)$ was rigidly held at its ends and a cylindrical support of diameter $1 \mathrm{~mm}$ was placed at the center. The sample was compressed to a length $\mathrm{L}$ $=55 \mathrm{~mm}$ to create a distorted arch similar to the schematic in Figure 3a. The section that is arched upward is irradiated using the $375 \mathrm{~nm}$ UV laser centered at a distance of L/4 from the rigid support. The photomechanical deformation is initially quasistatic resulting from the trans-cis isomerization (Figure $3 b$ ) until the edge of instability is reached. Then, the arch is found to deform to the second bifurcated state in an ultrafast manner. High-speed imaging reveals this actuation to also occur in $\sim 10 \mathrm{~ms}$ (see Supplementary Movie 3). Drawing from the idea in Figure $1 \mathrm{~b}$, the irradiated surface is exposed to $>500 \mathrm{~nm}$ light from a filtered halogen lamp for 300 seconds to induce the cis-trans back reaction. This restores the actuator's photoresponsiveness. After the ultrafast transition, the portion that was hitherto arched 
downward is now up. The reversible actuation now requires irradiating the part that is now arched upward, by simply moving the light source to a point that is at a distance of L/4 from the corresponding rigid support. Irradiation from the same direction can now lead to repeated back-and-forth actuation. The energy release during the actuation was computed using an approach similar to that used earlier. For the actuation illustrated in Figure 3b, the energy release and the specific power were found to be $3.3 \mathrm{~nJ}$ and $0.5 \mathrm{~kW} / \mathrm{m}^{3}$, respectively. Models are being evolved to understand how the energy release in this configuration compares against that in the simple bistable arch illustrated in Figure 1.

\subsection{Photomechanical control of the latency of actuation}

Complementing the strategy for repetitive actuation, we also explore routes for accelerating the approach to instability for the onset of snap-through. The approach to instability is quasistatic and is the rate-determining step for triggering the transition between the states. One approach for controlling this latency revolves around the utilization of articulated irradiation with $445 \mathrm{~nm}$ light, which is known to produce photo-strains via the trans-cis-trans reorientation (Weigert effect). These strains are more persistent than that achieved with UV irradiation. In an illustrative experiment, we compared azo-polyimide samples held in a contilever configuration and irradiated using UV and 445nm lasers polarized along the long-axis of the sample. Both samples deformed towards the actinic light. However, when the light is turned off, the $445 \mathrm{~nm}$-irradiated sample showed greater persistence of the photomechanical strains (Figure S2). To utilize this persistence, archshaped samples were irradiated to achieve a geometry that is perturbed and closer to the edge of instability. The greater persistence of the photo-strains with $445 \mathrm{~nm}$, which is found to be a function of the backbone rigidity of the polyimides, allows the actuator to remain "primed" closer to the instability, even when the light is turned off. When actuation is desired, much shorter length of irradiation is sufficient to trigger snap-through. The second, short irradiation 
from the primed state can be with either UV or $445 \mathrm{~nm}$ (which we use here, for illustrative purposes and experimental ease - the ideas are applicable for UV without loss of generality).

Figure 4a illustrates this approach. Initially, we irradiate for 5.1 seconds with linear polarized $445 \mathrm{~nm}$ light with intensity of $25 \mathrm{~mW} / \mathrm{cm}^{2}$, and the sample is deformed into a shape that is closer to the edge of instability. After switching off the irradiation, the sample (predominantly) remains in the deformed state. After about 15.0 seconds of no irradiation, the sample is irradiated again, and undergoes ultrafast snap-through in 2.4 seconds (see Supplementary Movie 4). To quantify the observations, the photo-generated strain was calculated. The local curvature of the film was calculated from the images. The change in curvature was calculated along the length of the sample as $\delta \kappa(x)=\kappa_{c}-\kappa_{a}$ where $\kappa_{c}$ is the local curvature of the deformed shape, and $\kappa_{a}$ is the local curvature of the initial shape. Figure S3 shows the photo-generated change in curvature with progressive irradiation at three different configurations. To quantify the evolution of the geometry, the average curvature range in the irradiated region of the film is given as: $\delta \kappa_{a v}=\frac{\int \delta \kappa(x) d x}{L_{i r r}}$. The integral is calculated over the irradiated length, and $L_{i r r}$ is the total irradiated length. Figure 4a shows the average curvature in the irradiated region as a function of time.

Curvature measurements show a small but measurable relaxation of the sample away from the edge of instability after the priming irradiation is turned off. The curvature relaxation is traceable to the loss of photo-induced strains, which is a function of the macromolecular structure and the rigidity of the backbone ${ }^{9}$. This relaxation of the strains in the azofunctionalized polyimide moves the actuator away from the edge of instability. To show that this relaxation is not an artifact of the arch-shaped geometry, experiments were also performed with cantilevers of the polyimide. Strips $\left(L_{0}=5 \mathrm{~mm}, W=1 \mathrm{~mm}, T=15 \mu \mathrm{m}\right)$ that were clamped at one end were irradiated with a $445 \mathrm{~nm}$ laser, also at an intensity of 25 $\mathrm{mW} / \mathrm{cm}^{2}$. The entire length of the cantilever is irradiated using linearly polarized light with 
the polarization set parallel to the long-axis and the average curvature is measured over the cantilever length. The evolution of the geometry is recorded as a function of time during irradiation and during post-illumination relaxation.

Figure $4 \mathrm{~b}$ shows the evolution of the curvature relaxation, as a function of time, after being irradiated for different time intervals. Longer irradiation leads to a larger prior curvature. Hence, the evolution of the geometry is characterized by normalizing the curvature with respect to the curvature at the end of irradiation, after which relaxation begins. The temporal evolution of the curvature is captured by fitting a biexponential model that explains the relaxation dynamics in terms of two characteristic time constant $\tau_{1}$ and $\tau_{2}$ as $\frac{\kappa}{\kappa_{o}}=$ $C_{1} \exp \left(-\frac{t}{\tau_{1}}\right)+C_{2} \exp \left(-\frac{t}{\tau_{2}}\right)^{20}$. After fitting the experimental data with the biexponential model a constant ratio of $\mathrm{C}_{1} / \mathrm{C}_{2}=0.35$ was obtained for each induced strain level prior to relaxation. $\tau_{1}$ and $\tau_{2}$ are found to vary as $\tau_{1}=2.1 * 10^{5} \kappa_{0}-1.3 * 10^{2}$ and $\tau_{2}=4.1 *$ $10^{6} \kappa_{0}-2.7 * 10^{3}$

This characterization offers a tool for modulating control strategies to achieve a piecewise approach to the snap-through instability by using irradiation in articulated intervals. One irradiation step to photomechanically pinch the sample near the edge of instability and the second to induce the snap-through in short order, when desired - while accounting for the relaxation that is outlined in Figure $4 \mathrm{~b}$. Dependence of the relaxation of photo-induced deformation on the prior level of irradiation/photomechanical curvature holds implication for utilization of these materials in responsive structures that exploit shape memory phenomena. In addition to placing bounds on the utilization of articulated irradiation as a route for modulating the mechanical behavior in bistable actuators, this measurement also points to non-linearities in the restoring mechanisms that lead to loss of photo-induced strains. 


\subsection{Geometrically prebiasing for snap-through}

A complementary strategy that can be seamlessly integrated with the aforementioned approaches involves the use of geometric or mechanical priming. Here we present an arch fabricated from a sample with a non-uniform cross section. By employing arches with nonuniform cross-sectional properties we break the symmetry of the arch and pre-bias it toward asymmetric snap-through. This asymmetry is primarily due to the differences in the flexural rigidity of the different sections of the arch. We find that such actuators are still bistable but are mechanically pre-biased for undergoing snap-through. That is, the levels of photo-strains required for transitioning between the bistable states can be reduced by significant fractions through such mechanical design (See Supplementary Information). It is found that a sample composed of a cross-section that is stepped (or L-shaped) creates an arch that is asymmetric. Finite element simulations predict that the required photo-strains for inducing snap-through in this type of arch can be reduced by approximately $36 \%$ over a similar uniform arch (see supplementary text, Figure S4). Figure 5a illustrates this idea. Experimentally, the approach to snap-through instability in this geometry following irradiation was bench-marked against that in a uniform cross-section arch in our prior experiments. It is apparent from Figure 5a that $\sim 30 \%$ reductions in the time for inducement of ultrafast actuation can be achieved using the mechanical pre-biasing in non-uniform cross-section devices. The advantage of this approach for reducing the latency is that it is not dependent on material history and is identically functional for bidirectional actuation. The actuation after reaching the edge of instability is still ultrafast (see Supplementary Movie 5). Photo and geometric priming can be used individually or in combination to modulate the latency of the actuator prior to the onset of instability for switching between the states. Figure $5 \mathrm{~b}$ illustrates the simulations of the actuation mechanism for the L-shaped samples that coincides with the geometric evolution observed in imaging. The geometry of these samples in $5 \mathrm{~b}(\mathrm{i})$ is characteristically assymetric due to the varying cross-section. Irradiation at the middle of the arch leads to quasistatic 
deformation, which advances the geometry to the edge of instability in $5 \mathrm{~b}$ (iii). The computed modification of the strain field at the surface during this deformation is shown in Figure $5 b$ (ii). At the edge of instability, ultrafast actuation ensues to invert the geometry as illustrated in Figure $5 b(v i)$. The difference in the strain-fields just prior to the snap-through and after the actuation is shown in Figure $5 b(v)$.

\section{Conclusions}

In summary, we have shown that opportunities exist for achieving enhanced and innovative devices based on light driven binary structures including micro-positioners for binary robotics, customizable topography control, and active valves for microfluidics. Challenges remain in controlling photo-responsive devices due to the superposition of nonlinear phenomena influencing the material response. Herein we have highlighted several tactics, ranging from modulated light control to geometric design, for addressing challenges inherent in the reliable and repeated actuation of light-responsive structures. In the future, combinatorial designs using a variety of control strategies can emerge by drawing from these ideas to achieve repetitive back-and-forth actuation, as well as to modulate the approach to the ultrafast snap-through. Indeed the modularity of these binary structures lends itself to designing countless arrangements of integrated and cooperative components. Continued attention on the fundamental behaviors of photomechanical materials that influence efficient and predictable performance in device-like environments should result in exciting new designs for functionally enhanced devices. 


\section{Acknowledgements}

D.H.W., L.-S.T., A.A.S, S.C. M.L.S., J.B., M.R.S. and T.J.W. acknowledge the support of the Materials and Manufacturing Directorate and Office of Scientific Research of the Air Force

Research Laboratory. M.R.S. also acknowledges support from the National Science Foundation (grant \#1435489).

\section{References}

1 Minko, S., Responsive Polymer Materials: Design and Applications, 101, WileyBlackwell, 2006.

2 Taccola, S. et al., Toward a New Generation of Electrically Controllable Hygromorphic Soft Actuators, Adv Mater., 2015, 27, 1668-1675.

3 Brochu, P. and Pei, Q., Advances in Dielectric Elastomers for Actuators and Artificial Muscles. Macromolecular Rapid Communications, 2010, 31, 10-36.

4 Yu, Y., Nakano, M. and Ikeda, T. Directed bending of a polymer film by light. Nature, 2003, 425, 145.

5 Finkelmann, H., Nishikawa, E., Pereira, G. and Warner, M. Phys. Rev. Lett., 2001, 87, 015501.

6 Wie, J. J., Lee, K. M., Smith, M. L., Vaia, R. a. and White, T. J., Soft Matter, 2013, 9, 9303.

7 Lee, K. M., Bunning, T. J. and White, T. J., Adv. Mater., 2012, 24, 2839.

8 Yu, Y., Nakano, M., Shishido, A., Shiono, T. and Ikeda, T., chem. Mater., 2004, 16, 1637.

9 Wang, D. H. et al., Macromolecules, 2011, 44, 3840.

10 Lee, K. M. et al., Adv. Funct. Mater., 2011, 21, 2913.

11 Lee, K. M., Tabiryan, N. V., Bunning, T. J. and White, T. J., J. Mater.Chem., 2012, 22, 691 .

12 Chen, M. et al., Photodeformable CLCP material: study on photo-activated microvalve applications, Appl. Phys. A, 2010, 102, 667-672.

13 Chen, M. et al., Photodeformable polymer material: towards light-driven micropump applications, Appl. Phys. A, 2010, 100, 39-43.

14 Yamada, M. et al., Photomobile Polymer Materials: Towards Light-Driven Plastic Motors, Angew. Chem. Int. Ed., 2008, 47, 4986-4988.

15 Cheng, F., Yin, R., Zhang, Y., Yen, C.-C. and Yu, Y., Fully plastic microrobots which manipulate objects using only visible light, Soft Matter, 2010, 6, 3447.

16 Shankar, M. R. et al., Proc. Natl. Acad. Sci.,2013, 110, 18792.

17 Wie, J. J., Wang, D. H., Lee, K. M., Tan, L.-S. and White, T. J., Molecular Engineering of Azobenzene-Functionalized Polyimides To Enhance Both Photomechanical Work and Motion, Chem. Mater., 2014, 26, 5223-5230.

18 Hafez, M., Litcher, M. D. and Dubowsky, S. IEEE/ASME Trans. Mechatronics, 2003, 8, 18 .

19 Shan, S. et al., Multistable Architected Materials for Trapping Elastic Strain Energy, Adv. Mater., 2015, 27, 4296-4301.

20 Dureiko, R. D., Schuele, D. E. and Singer, K. D., Modeling relaxation processes in poled electro-optic polymer films, J. Opt. Soc. Am. B, 1998, 15, 338. 
Figure 1: A buckled arch fabricated from an azobenzene-functionalized polyimide is utilized as a bistable actuator. a) A strip of length Lo that is rigidly held at its ends and compressed to a length L creates the buckled arch. b) Multiplexed irradiation loop for triggering repetitive snap-through cycles. c) Variation of latency time $\left(t_{c r}\right)$ to trigger snap-through as a function of intensity of the incident $375 \mathrm{~nm}$ UV. d) Normalized time to actuation of the actuator shown in figure $1 \mathrm{~b}$ for multiple cycles of irradiation/relaxation.

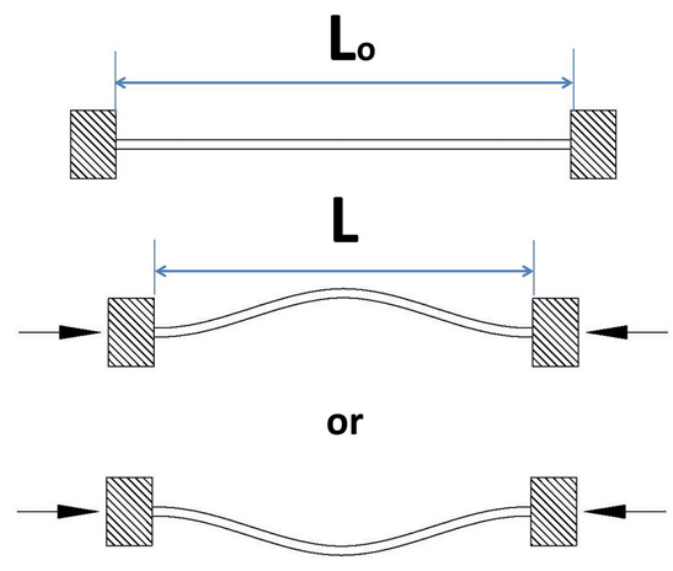

Figure-1-a(Shankar) 


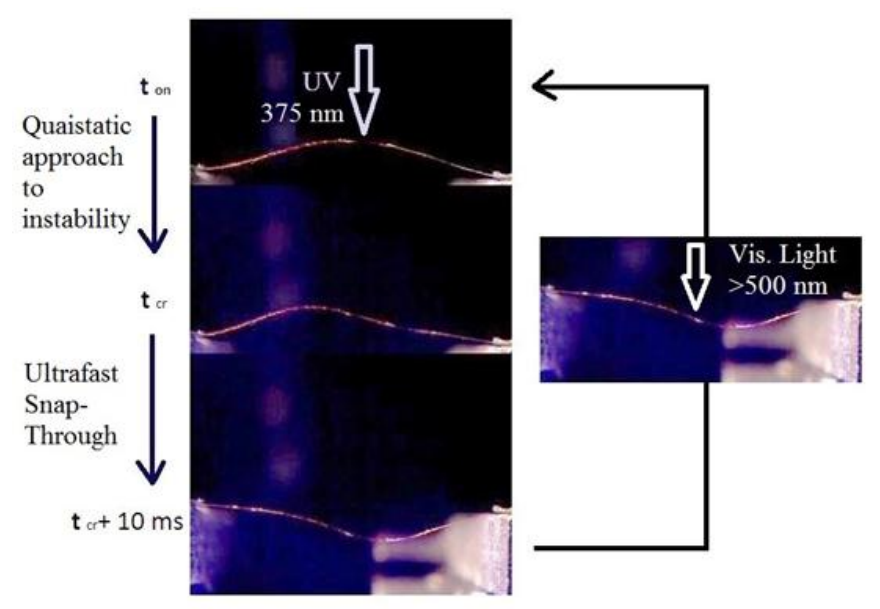

Figure-1-b(Shankar)

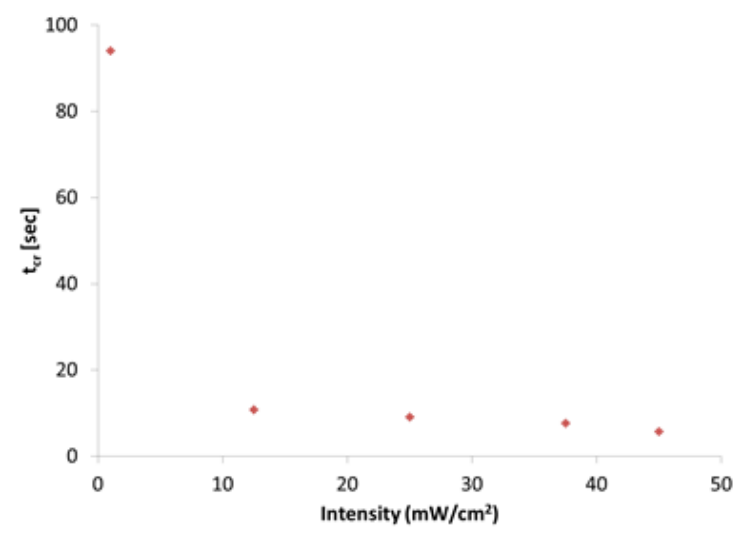

Figure-1-c(Shankar)

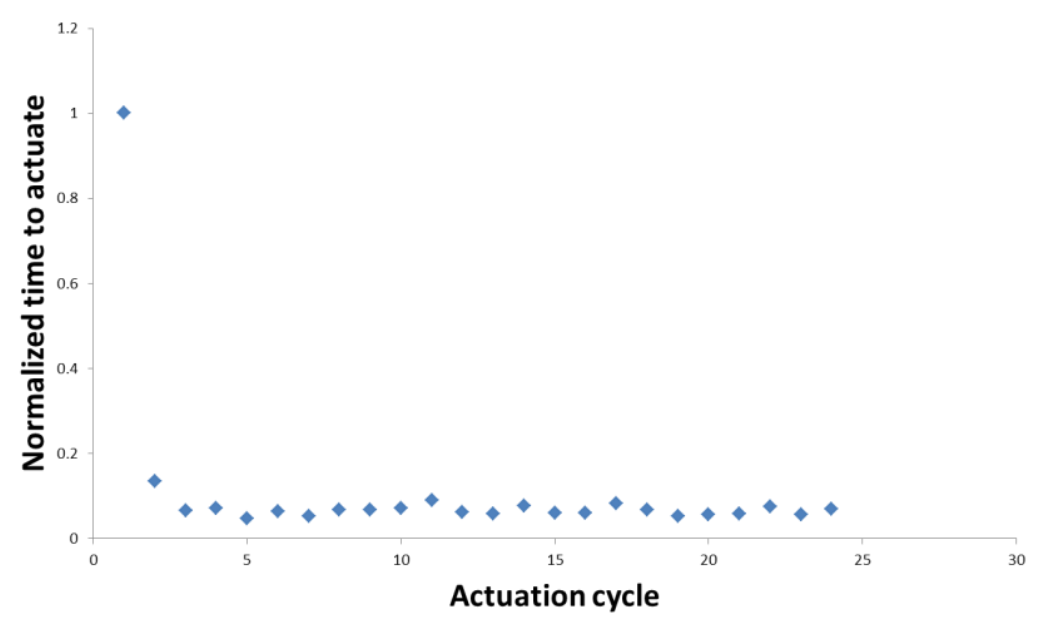

Figure 1-d(Shankar) 
Figure 2. Integration of actuator arrays to fabricate prototypical devices. a) 3x3 planar array of actuators to demonstrate customizable topographies. Letters P, I and T are spelled by toggling the actuators appropriately. The actuators that are arched upward are marked green and those arched downward are marked red. b) A tripod-like device that can be used as a unitcell of a binary robot. Toggling the actuators reorients the normal vector of the "table" that rests on them.
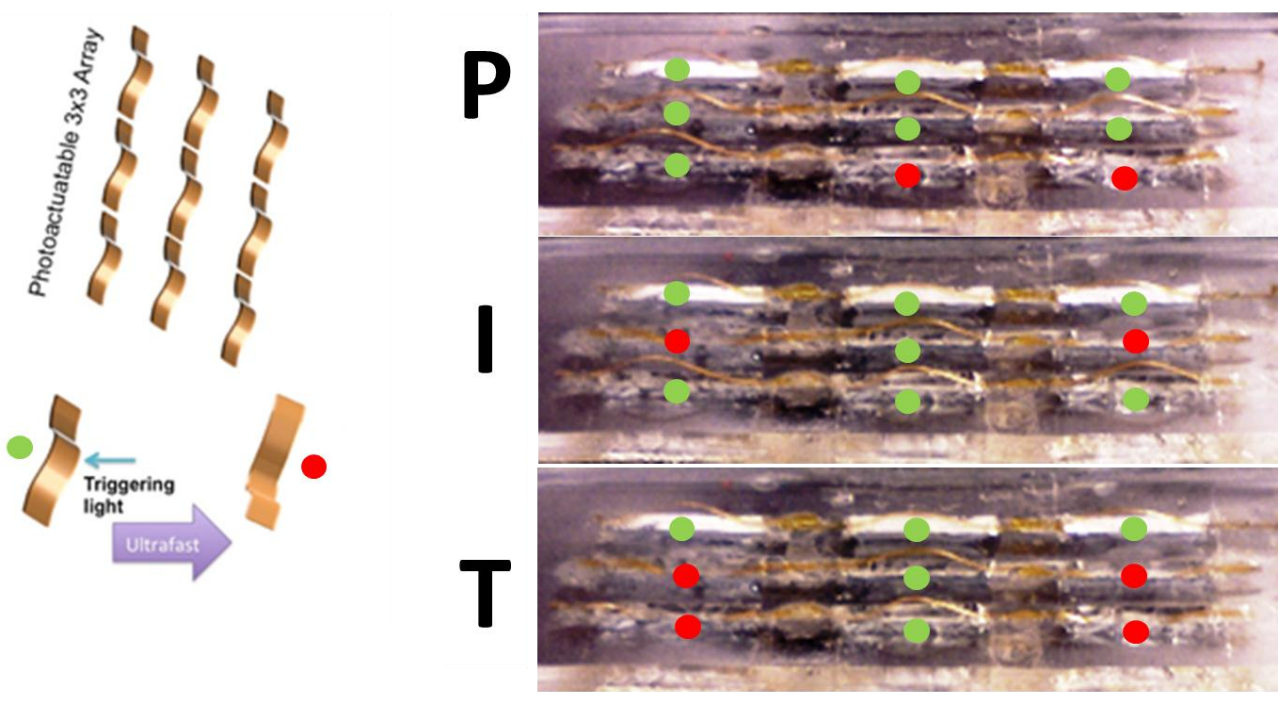

Figure 2-a(Shankar) 

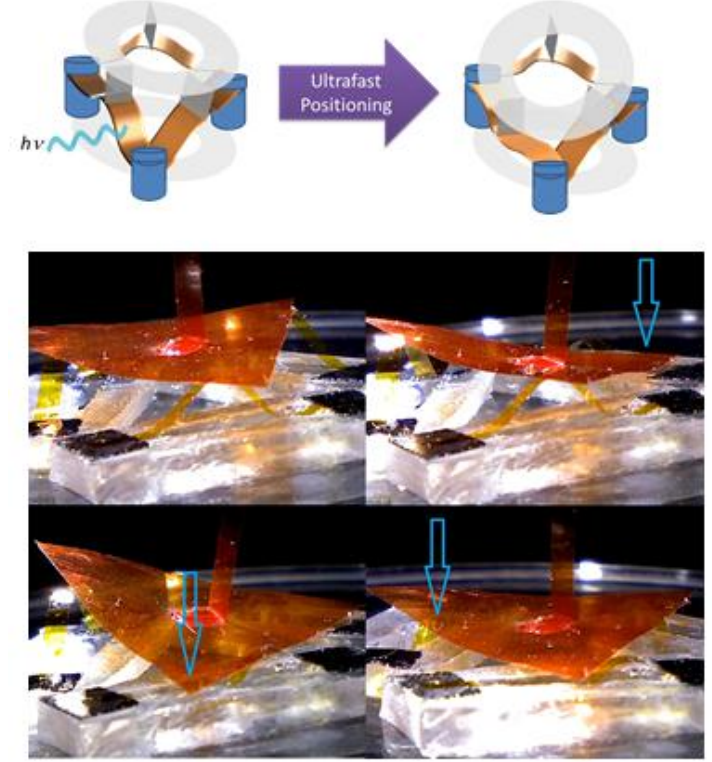

Figure-2-b(Shankar) 
Figure 3. Back and forth actuation with unidirectional irradiation. a) A "continuous beam" is rigidly held at the ends and deformed by a cylindrical support placed midway between the ends. b) Irradiation control loop for triggering multiple snap-through cycles in azobenzenefunctionalized polyimide.

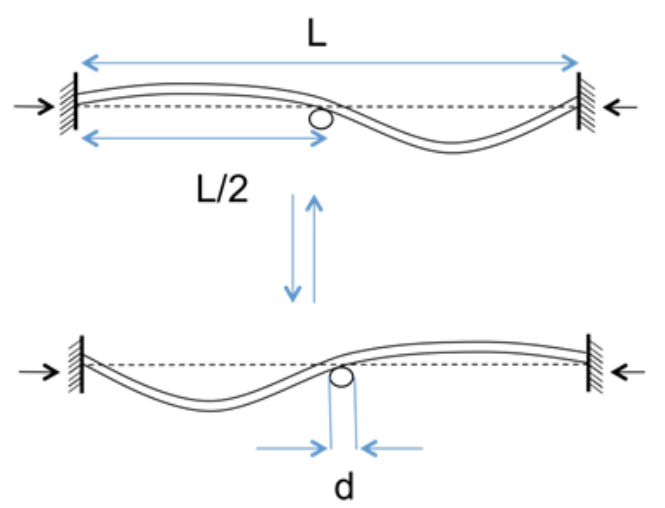

Figure-3-a(Shankar)

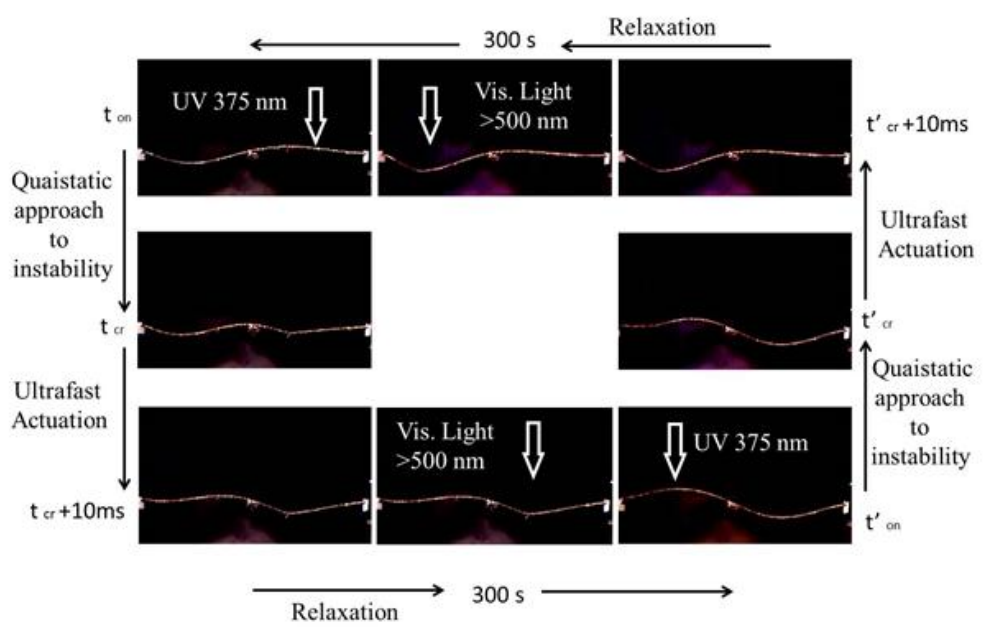

Figure-3-b(Shankar) 
Figure 4. Controlling the approach to snap-through using articulated irradiation. a) Average curvature $\left(\delta \kappa_{\mathrm{av}}\right)$ in the irradiated zone following initial irradiation to prime the sample closer to the edge of instability, relaxation of the sample away from the edge of instability after irradiation is stopped and onset of snap-through after a short second irradiation is shown. b) Evolution of the geometry of a cantilever as a function of time after irradiation for various intervals of time $(\mathrm{t})$.
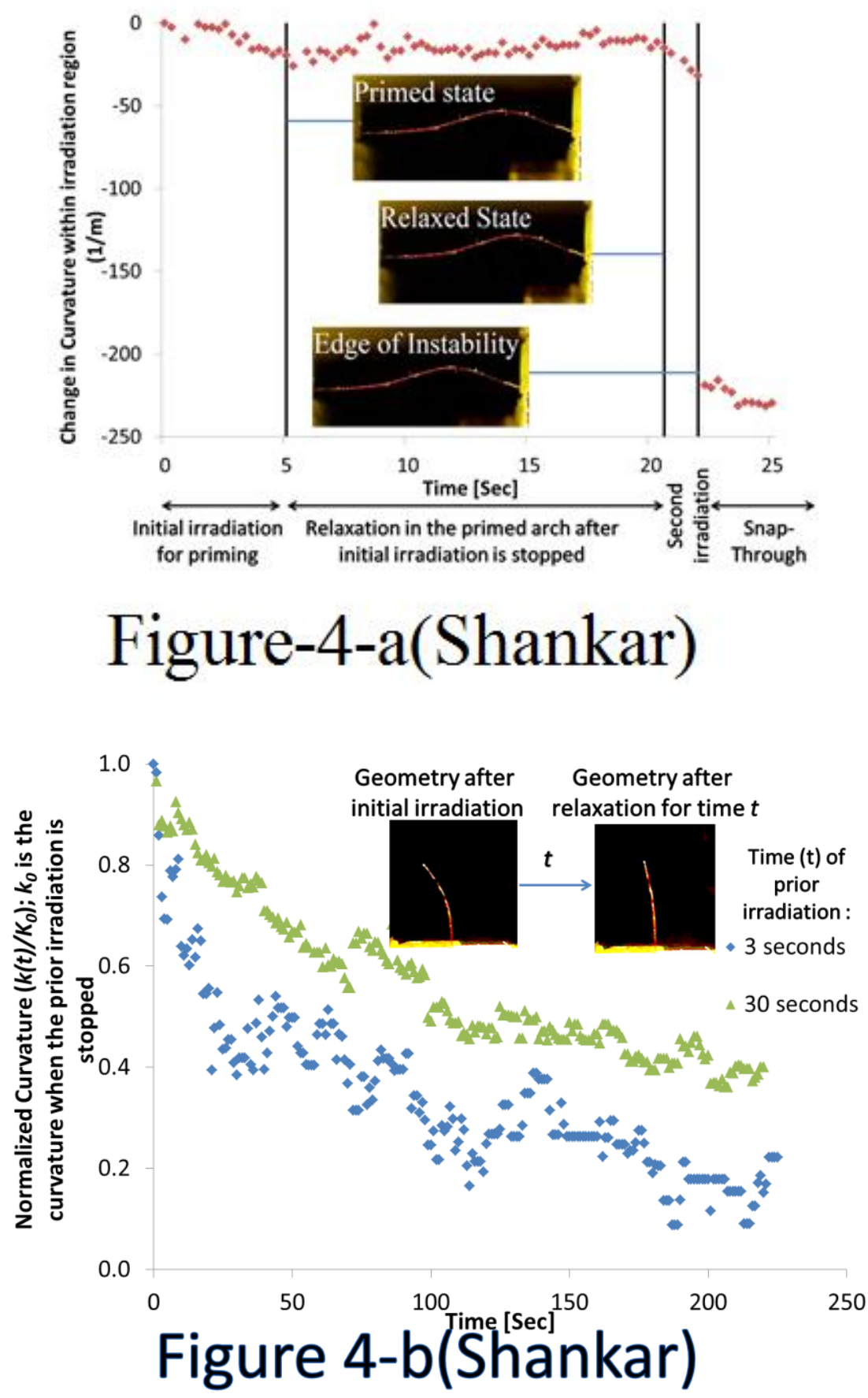
Figure 5. Accelerating the approach to instability via mechanical design a) Time ( $\left.t_{c r}\right)$ for inducing snap-through in L-shaped (stepped) samples vs. those in samples of uniform crosssection ( $1 \mathrm{~mm}$ wide). In the L-shaped samples, the width of the sample is $1.5 \mathrm{~mm}$ on the left and $0.75 \mathrm{~mm}$ on the right. The thickness of the sample is $15 \mu \mathrm{m}$ throughout the length. b) Finite element simulation results showing side views of configurations of the L-shaped arch and strain differences between states. The horizontal and vertical coordinates of material points are given by $r_{1}$ and $r_{2}$, respectively. All units are in millimeters except for the strains, which are unitless. $L_{o}=15 \mathrm{~mm}$ and the sample is compressed by $L_{o}-L=\Delta L=48 \mu \mathrm{m}$. i) The initial buckled configuration of the arch is asymmetric. The peak point of the arch is located at approximately $8 \mathrm{~mm}$ from the left end instead of approximately $7.25 \mathrm{~mm}$. ii) The computed strain difference at the irradiated surface between the initial buckled state and the point of instability. iii) and iv) The point of elastic instability right before snap-through commences. v) The computed strain difference at the irradiated surface between the point of instability and the state just after snap-through. vi) The arch configuration just after snap-through. Strain differences were computed by subtracting the previous state from the following state. 


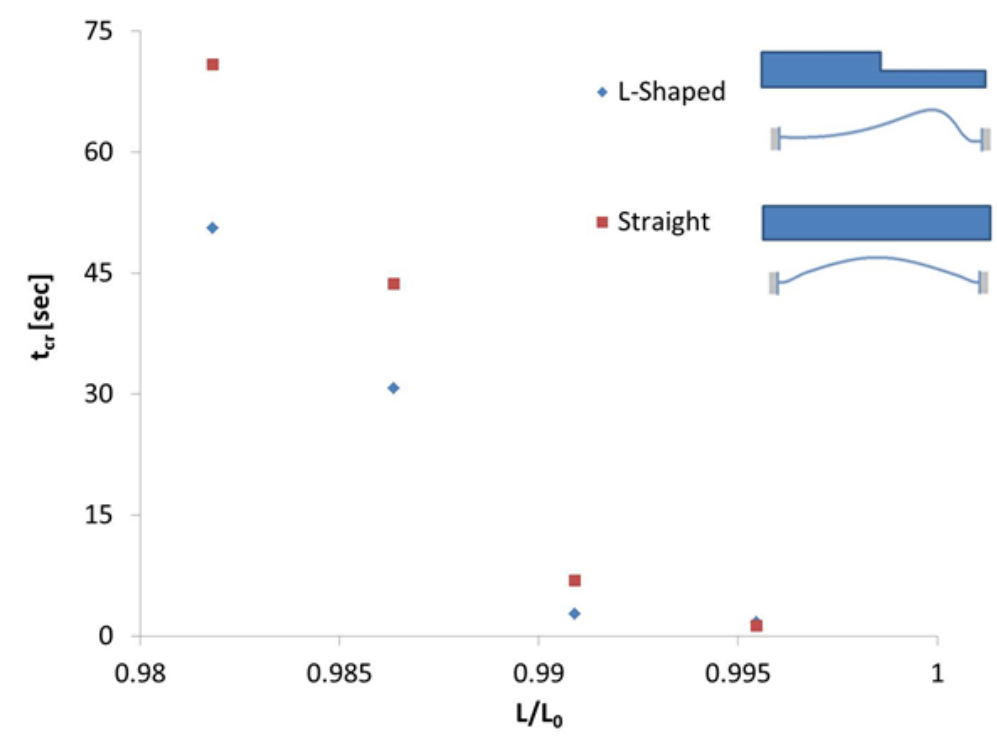

Figure-5-a(Shankar)

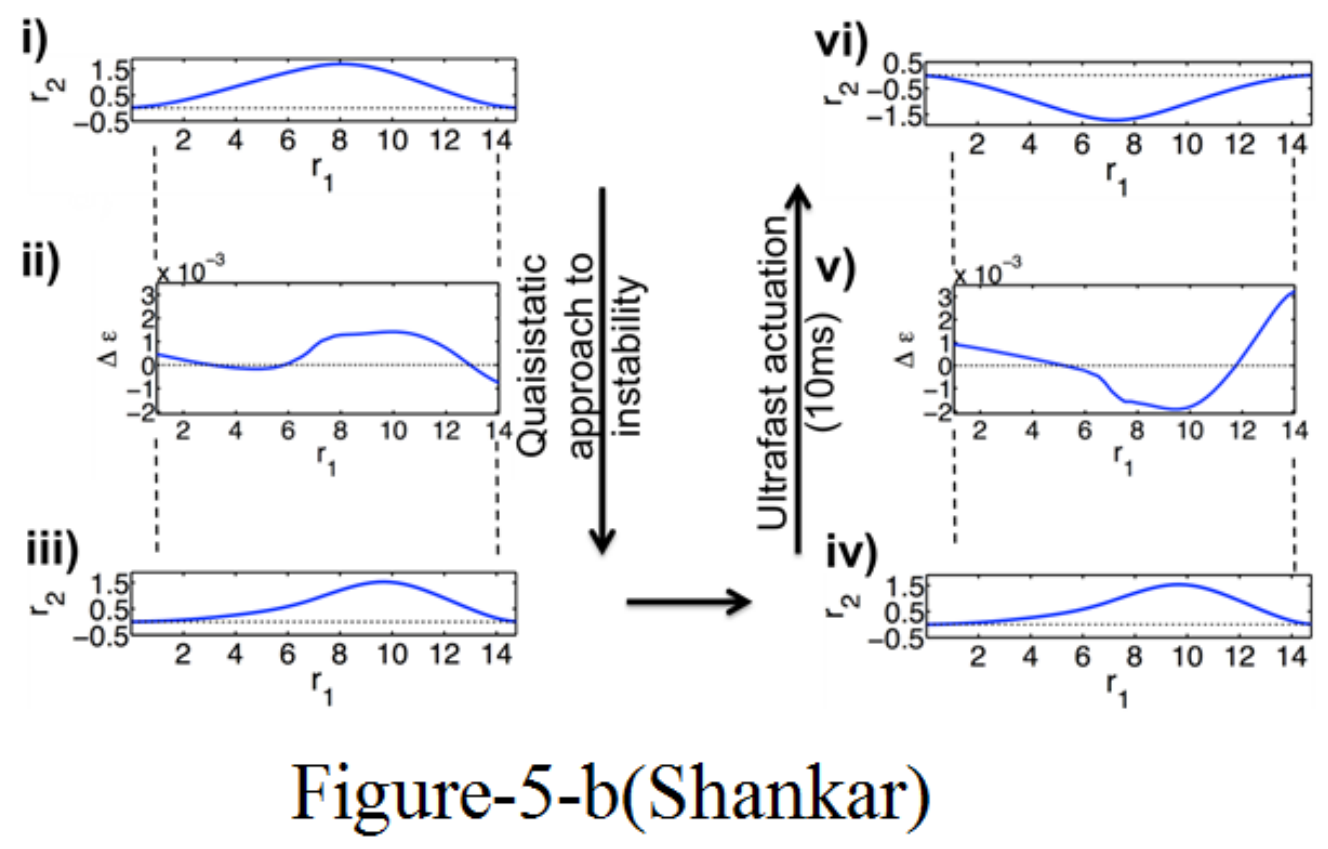

